Research Article

\title{
Performance of Polar-Coded 3D Image Transmission over Fading Channel
}

\author{
Jianjun Hao, ${ }^{1}$ Luyao Liu, ${ }^{1}$ and Wei Chen $\mathbb{1 D}^{2,3}$ \\ ${ }^{1}$ School of Electronic and Information Engineering, Shandong University of Science \& Technology, Qingdao, \\ Shandong 266590, China \\ ${ }^{2}$ School of Computer Science and Technology, Mine Digitization Engineering Research Center of the Ministry of Education, \\ China University of Mining and Technology, Xuzhou, Jiangsu 221116, China \\ ${ }^{3}$ School of Earth and Space Sciences, Peking University, Beijing 100871, China \\ Correspondence should be addressed to Wei Chen; chenwdavior@163.com
}

Received 18 April 2020; Revised 20 May 2020; Accepted 27 May 2020; Published 16 June 2020

Guest Editor: Chi-Hua Chen

Copyright (c) 2020 Jianjun Hao et al. This is an open access article distributed under the Creative Commons Attribution License, which permits unrestricted use, distribution, and reproduction in any medium, provided the original work is properly cited.

Any signal transmitted over an air-to-ground channel is corrupted by fading, noise, and interference. In this paper, a Polar-coded 3D point cloud image transmission system with fading channel is modeled, and also the simulation is performed to verify its performance in terms of 3D point cloud image data transmission over Rician channel with Gaussian white noise and overlap of Gaussian white noise + periodic pulse jamming separately. The comparison of Polar-coded scheme with RS-coded scheme in the same scenario indicates that Polar-coded system gives far better performance against AWGN noise and fading than the RS-coded system does in the case of short block length. But RS-coded scheme shows better performance on antipulse jamming than that of Polar-coded scheme, while there is no interleaving between codewords.

\section{Introduction}

Unmanned Aerial Vehicles (UAV) communication systems are more accessible to be applied in the civilian and military domains, such as building survey, target reconnaissance, surface mapping, precision agriculture, and postdisaster rescue. In these applications, it is often necessary to capture target images (videos) or 3D images $[1,2]$. For example, in $3 \mathrm{D}$ detection of military targets, these $3 \mathrm{D}$ images need to be sent back to the ground station in real time, thus to guide the flight path or attitude adjusting by the drone operator. While transmitting on air-to-ground channel, these 3D images (videos) suffer from fading, noises, and interferences (such as radar signal jamming and a periodic strong pulse jamming) [3-5], and these will inevitably lead to bit errors at the receiver. A certain number of errors will cause changes in the shape and color of the $3 \mathrm{D}$ object and degrade the image quality.

Channel code can effectively reduce the transmission error rate and ensure reliable transmission against hostile channel conditions. Therefore, the application of channel code in a data transmission system can mitigate the effects of channel fading and noise interference, so as to improve the reliability of data transmission. In recent years, Polar code has been recognized and employed due to its good characteristics. In 2016, 3GPP was announced to adopt Polar code as the control channel code scheme for 5G mobile communication in eMBB scenario.

Polar code is a new channel coding method proposed by Erdal Arikan of Bilkent University, Turkey, in 2007. It is currently the only channel coding method which can theoretically reach the Shannon channel capacity. Compared with LPDC codes, Polar code scheme can achieve reliable communication with lower coding and decoding complexity.

Recent studies in Polar code employed in voice and image transmission achieved some valuable results. A voice transmission simulation adopting Polar code over AWGN and Rayleigh channel is performed in lecture [6], and the results show that this scheme provides high performance in 
bit error correction. In [7], the transmission performance of Polar-coded and OFDM-modulated grayscale images is tested over AWGN channel; the results show that both PSNR and structural similarity (SSIM) are superior to the $\mathrm{BCH}$ code scheme in BER. In [8], the authors simulated Polar-coded image by passing through Rayleigh fading transmission system to test its performance on antifading. Zhao Shengmei and her team of Nanjing University of Posts and Telecommunications adopt combined Polar coding with OFDM scheme to test its performance on transmitting grayscale image over Rician fading channel. They drew a conclusion that, under the condition of the same code rate and code length, PSNR of received image can be improved by $4 \mathrm{~dB}$ compared to the LDPC coding scheme [9]. In [10], an unequal error protection scheme is proposed to alleviate the distortion of received image caused by transmission bit errors. The simulation results show that it provides $7 \mathrm{~dB}$ gain compared to the unsuitable UEP scheme. In lecture [11], a chaotic interleaving technique is proposed for improving a transmission of colored images over burst error environment through merging it with error control scheme, but this advantage is achieved at expense of complexity. In [12], the authors proposed a method to improve transmission speed of $3 \mathrm{D}$ point cloud by removing unnecessary information, but it achieved transmission rate improvement at the expense of reliability.

With the rise of VR (Virtual Reality), the processing and transmitting of 3D images and 3D video also attract many scholars' attention. Since data format and data structure of $3 \mathrm{D}$ images are quite different from $2 \mathrm{D}$ images, as well as the evaluation of image quality, transmitting $3 \mathrm{D}$ images becomes a big challenge. Aiming at solving the problem of transmitting images over mobile channels, we modeled a Polar-coded image transmission system with Rician fading channel to verify its performance on combating Gaussian noise and periodic strong pulse jamming and make a comparison with RS code scheme.

\section{Polar Coding}

The Polar-coding method is based on channel polarization which includes two processes: channel merging and channel splitting. $N$-independent binary symmetric discrete memoryless channels (BDMC) $W$ can be regarded as a new joint channel $W_{N}$; we call this channel combining. After $N$ original channels linearly combined and a joint channel generated, then the joint channel split into many new channels according to a certain rule; hence, a phenomenon called "channel polarization" occurs [11]. After the channel splitting, capacity of some split channels is equal to 0 (fullnoise channel), and that of others is equal to 1 (noiseless channel), but the total channel capacity remains the same. According to this phenomenon, the data bits are then sent to transmitting over these noiseless channels $[13,14]$, so that the Shannon limit can be approached.

Polar codes can be encoded in the form of linear block codes. The mapping from the source code sequence $u_{1}^{N}$ to the transition sequence $x_{1}^{N}$ can be extracted and expressed by a matrix $x_{1}^{N}=u_{1}^{N} G_{N}$, and the original recursive equation of the generator matrix $G_{N}$ is given by

$$
G_{N}=\left(I_{N / 2} \otimes F^{\otimes n}\right) R_{N}\left(I_{2} \otimes G_{N / 2}\right),
$$

where $F^{\otimes n}=\left[\begin{array}{cc}F^{\otimes n-1} & 0 \\ F^{\otimes n-1} & F^{\otimes n-1}\end{array}\right]$ is generated by recursion of $F$, $F=\left[\begin{array}{ll}1 & 0 \\ 1 & 1\end{array}\right], I$ represents the identity matrix, and $\otimes$ is the Kronecker product; when $A_{N}=\left(I_{N / 2} \otimes F\right) R_{N}$, equation (1) becomes $A_{4}=A_{N}\left(I_{2} \otimes G_{N / 2}\right)$. The recursive construction of the generator matrix can be implemented by generating $A_{N}$. For instance, given $N=8$, we obtain

$$
A_{8}=\left[\begin{array}{llllllll}
1 & 0 & 0 & 0 & 0 & 0 & 0 & 0 \\
1 & 0 & 0 & 0 & 1 & 0 & 0 & 0 \\
0 & 1 & 0 & 0 & 0 & 0 & 0 & 0 \\
0 & 1 & 0 & 0 & 0 & 1 & 0 & 0 \\
0 & 0 & 1 & 0 & 0 & 0 & 0 & 0 \\
0 & 0 & 1 & 0 & 0 & 0 & 1 & 0 \\
0 & 0 & 0 & 1 & 0 & 0 & 0 & 0 \\
0 & 0 & 0 & 1 & 0 & 0 & 0 & 1
\end{array}\right] .
$$

There are two parameters for the channels mentioned above, symmetric capacity $I(W)$ and the Bhattacharyya parameters $Z(W) . I(W)$ is the maximum information transmission rate of the channel, and $Z(W)$ is the minimum transmission error probability. One has

$$
\begin{aligned}
& I(W)=\sum_{y \in Y} \sum_{x \in X} \frac{1}{2} W\left(\frac{y}{x}\right) l b \frac{W(y / x)}{(1 / 2) W(y / 0)+(1 / 2) W(y / 1)} \\
& Z(W)=\sum_{y \in Y} \sqrt{W\left(\frac{y}{0}\right) W\left(\frac{y}{1}\right)},
\end{aligned}
$$

where $W(y / x)$ is the transition probability $X \longrightarrow Y$. After channel splitting, the capacity values of these channels are subjected to "0-1" distribution. Polarization coding is to select those channels with full channel capacity. $A$ is defined as the selected channels' set for transmitting information data; $K$ is the size of set $A$ which represents the number of transmitted information data bits. Correspondingly, $A^{C}$ is the set of channels employed to transmit the known bits; hence, the mapping code $x_{1}^{N}=u_{1}^{N} G_{N}$ can be decomposed into

$$
x_{1}^{N}=u_{A} G_{N}(A) \oplus u_{A^{C}} G_{N}\left(A^{C}\right),
$$

where $G_{N}(A)$ is equivalent to the information channel vector selected from $G_{N}$ and $G_{N}\left(A^{C}\right)$ is the junk channel matrix composed of the remaining $G_{N}$ rows, which determines the elements used to select the channel in the index set $A . u_{A^{C}}$ is the default bit sequence value determined by the information source and destination [15]. Once the source message is given, the Polar code block $x_{1}^{N}$ can be obtained by 
constructing a set of $\left(N, K, A, u_{A^{C}}\right)$. The process of polarization coding is as follows:

(1) Assuming that the coding length is $N=2^{n}, n>1$, calculate the generator matrix recursively according to formula $G_{N}=A_{N}\left(I_{2} \otimes G_{N / 2}\right)$

(2) Set the coding efficiency value $R$ and select the method of calculating the Bhattacharyya parameters under the BEC channel condition to obtain the full channel index set $A$

(3) Obtain generator matrix and channel index set; then select channels by these channel indexes to determine $G_{N}(A)$

(4) The sender and receiver agree in advance on the default bit sequence $u_{A^{C}}$ and send it to the junk channel $G_{N}\left(A^{\mathrm{c}}\right)$ for transmission

(5) After determining the set $\left(N, K, A, u_{A^{C}}\right)$ in accordance with the previous four steps, calculate the coding sequence $x_{1}^{N}$ to implement the polarization encoding

In this article, we use CRC-SCL decoding method to recover the image. The CRC-SCL decoding algorithm is based on the SCL decoding scheme by adding a cyclic redundancy check CRC block.

The process of CRC-SCL decoding algorithm is as follows: after the source generates $K$-bit information sequence, it is first sent to the CRC encoder to get m-bit check bit to attach to $K$ bit information sequence; the obtained $k+m$ bits data block is then sent to the Polar encoder with a code length of $N$ and a code rate of $(k-m) / N$; at the receiver, the received block first passes the SCL decoder, and the survived $L$ decoding paths are verified by CRC check in order of large to small by metric norm. If the verification result is zero by CRC check, the path is considered to be correct and output. If no path passes the verification, the CRC-SCL algorithm is degraded to SCL algorithm.

\section{Polar-Coded Image Transmission System Model}

The image transmission system modeled in this paper adopts Polar code as the channel coding method and QPSK as modulation scheme. The bit rate is supposed to be $10 \mathrm{Mbps}$, carrier frequency $5 \mathrm{GHz}$, and total link power loss $20 \mathrm{~dB}$. Considering that there is often a light sight propagation path between UAV and ground, we choose Rician fading channel with 3 propagation paths (including a direct path and 2 reflect paths with time delay $0.1 \mu$ s and $0.3 \mu$ s individually) as the wireless channel model. The noise is additive Gaussian white noise, and we use periodic strong jamming pulse (more than $5 \mathrm{~dB}$ higher than signal in mean power within jamming pulse duration) as pulse interference. The jamming pulse is presented as

$$
J(t)=\eta_{i} \sum\left[g(t+n T)-g\left(t+n T+T_{c}\right)\right] e^{j 2 \pi f_{0}\left(t+\tau_{i}\right)},
$$

where $\eta_{i}$ is the amplitude of strong jamming pulse which follows Gaussian distribution and the random time delay
$\operatorname{MSE}(a, b)=(1 /(N \times M)) \sum_{x=0}^{N-1} \sum_{y=0}^{M-1}[a(x, y)-b(x, y)]^{2}$ follows uniform distribution. $T$ is the pulse width, and $\operatorname{PSNR}(a, b)=10 \lg \left[255^{2} / \operatorname{MSE}(a, b)\right]$ denotes the period of jamming pulse. $f_{0}$ is the frequency of sine signal in jamming pulse duration, and $g(t)$ represents rectangular pulse. The image transmission system model is illustrated in Figure 1.

We established a 3D image transmission system model with Rician fading channel by MATLAB m-file. Polar code scheme with code length of 1024 and coding efficiency of $1 / 2$ is employed to encode the message under the condition of BEC channel with a deletion probability $p=0.5$ and 2 decoding paths. After being modulated by $\pi / 4$-QPSK, $2 \mathrm{D}$ image and 3D image signals transmit over a Rician fading channel with Gaussian noise, periodic strong pulse jamming, and Gaussian noise + periodic strong pulse jamming, respectively, to verify the performances of 3D image transmission. Also a RS-coded scheme (BM decoding) with coding efficiency 0.5 is performed over a Rician channel to compare with Polar-coded system in transmission performances.

In this article, the demonstration scheme is passing the $2 \mathrm{D}$ image data and the $3 \mathrm{D}$ point cloud image data through the established transmission system. The transmitted images are examined for their visual quality by using two different image quality assessment metrics, peak signal-to-noise ratio (PSNR, for 2D image) and point error ratio (PER, for 2D point cloud).

\section{Simulation of 2D Image Passing through the Polar-QPSK System}

In 2D image transmission simulation, a grayscale image Lena is subjected to Polar/RS encoding and then modulated by QPSK modulator; the output signals are sent over a Rician fading channel with Gaussian white noise to verify the performance against fading and Gaussian white noise.

Figures 2(a)-2(c) are the Polar-coded images reconstructed at receiver after passing through Polar/RS-coded transmission system with Rician fading channel $(k=4)$ and Gaussian noise. The received image and its PSNR under different SNR (average signal-to-noise ratio at the receiver) are shown in Figures 3(a)-3(c). The equation for calculating PSNR is

$$
\begin{aligned}
\operatorname{MSE}(a, b) & =\frac{1}{N \times M} \sum_{x=0}^{N-1} \sum_{y=0}^{M-1}[a(x, y)-b(x, y)]^{2}, \\
\operatorname{PSNR}(a, b) & =10 \lg \left[\frac{255^{2}}{\operatorname{MSE}(a, b)}\right] .
\end{aligned}
$$

In order to study the influence on image quality caused by pulse jamming, we use a nonfading channel to carry out the simulation. Figures 4(a) and 4(b) are the reconstructed Polar-coded images at receiver after passing through a channel without fading but interfered by periodic strong jamming pulse (with period of $1.64 \mathrm{~ms}$, i.e., 8 codewords' width). Figures 4(c) and 4(d) show the reconstructed RScoded images under same conditions. Here, JSR is the ratio of the average power of the jamming pulse to the average 


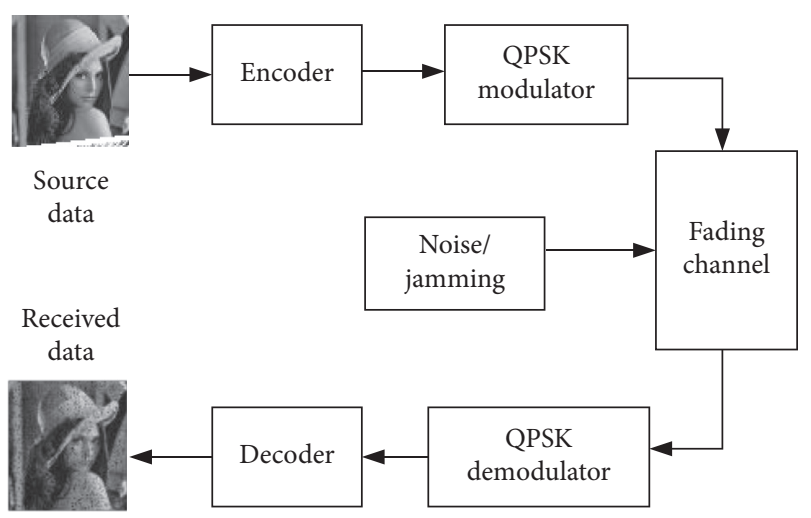

FIgURE 1: Polar-coded image transmission system model.

power of the signal in pulse duration, and the jamming pulse width is $12.8 \mu \mathrm{s}$ (64 symbols). For a strong jamming pulse (JSR $>4 \mathrm{~dB}$ ), jamming pulse amplitude's variation has a little effect on Polar-coded image or RS-coded image quality, as shown in Figure 4. Figures 4(a)-4(d) also indicate that RS code scheme provides a better performance on bursts error caused by strong pulse jamming than Polar code does.

Figures 5(a)-5(c) are the reconstructed Polar-coded images passed through the fading channel (Rician channel, $k=2$ ) with Gaussian white noise and periodic pulse jamming (jamming pulse width of $10.24 \mu \mathrm{s}$ ). It can be seen that while SNR increases, the image PSNR improves rapidly, but when SNR is up to a certain value ( $1 \mathrm{~dB}$ in this experiment), there is no longer evident evaluation in PSNR. Although the RS code has good antipulse jamming performance, it cannot achieve a high image quality at low SNR (Figures 6(a)-6(c)).

\section{Simulation of 3D Point Cloud Passing through the Polar-QPSK System}

In $3 \mathrm{D}$ image transmission performance verification experiment, we extract point cloud data from a 3D image file-teapot.obj, and then these image data are sent to pass through Polar-coded and RS-coded 3D image system, respectively.

Figure $7(a)$ is the original image in obj format, and Figure $7(\mathrm{~b})$ is the extracted $3 \mathrm{D}$ point cloud without color parameter.

In this paper, we use the point filling method to convert the extracted point cloud data to bit streams for transmission.

$3 \mathrm{D}$ point cloud image data are processed in steps as follows:

(1) Quantize coordinate value of the point to an integer; the $x$ coordinate range is from to 1 to $141, y$ from 1 to 70 , and $z$ from 1 to 86 .

(2) According to the value of $z$, the $3 \mathrm{D}$ point cloud is divided into 86 layers (planes). In each layer, those positions on each plane with a coordinate value are set to 1 , and the others are set to 0 ; thus, a $141 * 70 * 86$ $3 \mathrm{D}$ matrix with elements " 0 " or " 1 " is generated.
(3) Reconstruct this 3D matrix into a one-dimensional data sequence, thus generate a bit stream of 848820 bits, and hence fill the bit stream to a length of $2 n$ and send it to the encoder of the transmission system.

(4) At receiver after decoding the bit stream at the receiving end, the decoded sequence is then reconstructed into a 3-dimensional matrix of $141 * 70 * 86$. In each layer, when a bit is decided as 1 , it means there is a voxel at the corresponding position, and the value is taken out to reconstruct the 3D point cloud image.

Figures 8(a), 8(b), 9(a), and 9(b) are the reconstructed $3 \mathrm{D}$ point clouds after passing through Polar-coded and RS-coded system, respectively. The channel in transmission system adopted Rician fading channel $(k=2)$ with Gaussian white noise. Figure 10 shows the plot of point error ratio (PER) versus SNR; it can be seen that the Polarcoded cloud image quality improves rapidly with the increase of signal-to-noise ratio, whereas point error ratio curve of RS scheme depicts that there is no evident improvement in point cloud quality with SNR increase. Here, PER is the ratio of the amount of error points to total $141 * 70 * 86$ points. It indicates that the performance against fading and Gaussian white noise of Polarcoded system is evidently better than that of RS-coded system.

Figures 11(a)-11(d) and 12(a)-12(d) are the recovered $3 \mathrm{D}$ point clouds at the receiving end of Polar-coded system and RS-coded system, respectively.

Point clouds are also sent to pass through Polar/RS system with Rician fading channel $(k=2)$, Gaussian white noise, and periodic pulse jamming $(12.8 \mu$ s of pulse width, $5 \mathrm{~dB}$ of JSR). The amount of error points of total $141 * 70 * 86$ points denoted by $M$ in figures is the evaluation index of recovered image quality. Figures 11(a)-11(d) and Figure 13 illustrate that the quality of point cloud evaluates rapidly with the increase of the signal-to-noise ratio, but while SNR exceeds $2 \mathrm{~dB}$, the error points change very little with $\mathrm{SNR}$. When $\mathrm{SNR}=4$, the error points number $M$ is only 48 less than that of $\mathrm{SNR}=2$, and the amount of error points at SNR $=6$ is only 308 less than that of $\mathrm{SNR}=4$.

When $\mathrm{SNR}<4$, Polar-coded transmission system shows far better performance than that of RS coding system on combating fading, AWGN noise, and pulse jamming, but when SNR is up to 6, the error points of recovered teapot point cloud of RS-coded system decline to 30 , which means that its quality is very close to the original picture, as shown in Figure 13.

Figures 14(a), 14(b), 15(a), and 15(b) are the received 3D point clouds of Polar-coded scheme and RS-coded scheme via nonfading channels with periodic strong pulse interference, where Figures 14(a) and 15(a) are the received images interfered by a strong pulse with width of $12.8 \mu \mathrm{s}$ (64 symbols), and Figures 14(b) and 15(b) are the received images interfered by a strong pulse with width of $51.2 \mu \mathrm{s}$. As 


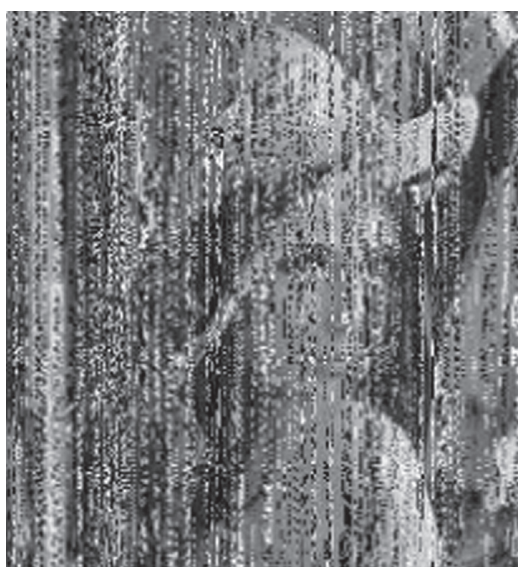

(a)

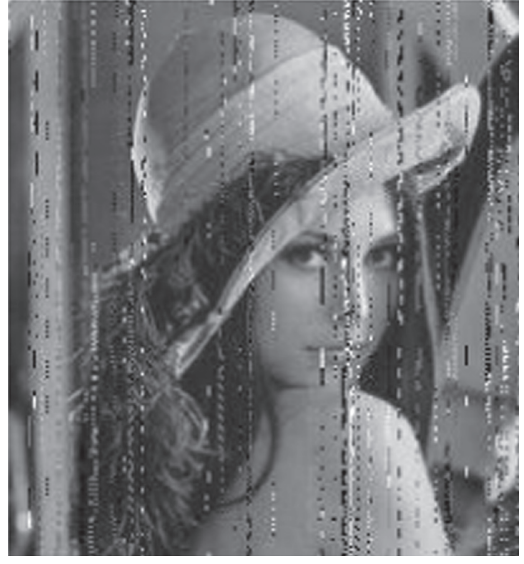

(b)

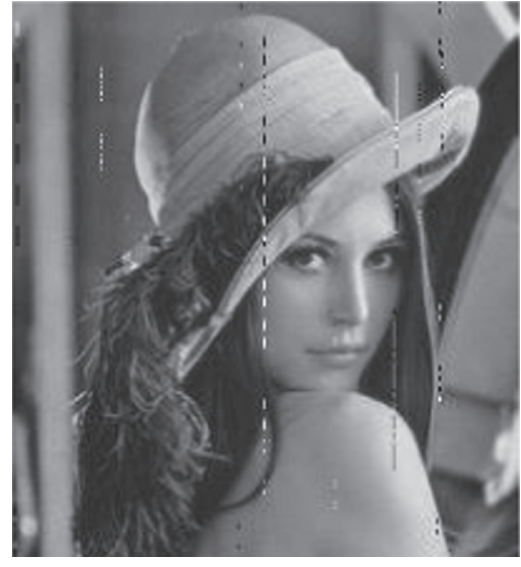

(c)

FIgURE 2: Recovered Polar-coded image (transmitted over Rician fading channel with Gaussian noise). (a) $S N R=-2 \mathrm{~dB}$; PSNR $=30.60$. (b) $\mathrm{SNR}=-1 \mathrm{~dB} ; \mathrm{PSNR}=37.09$. (c) $\mathrm{SNR}=0 \mathrm{~dB} ; \mathrm{PSNR}=46.63$.

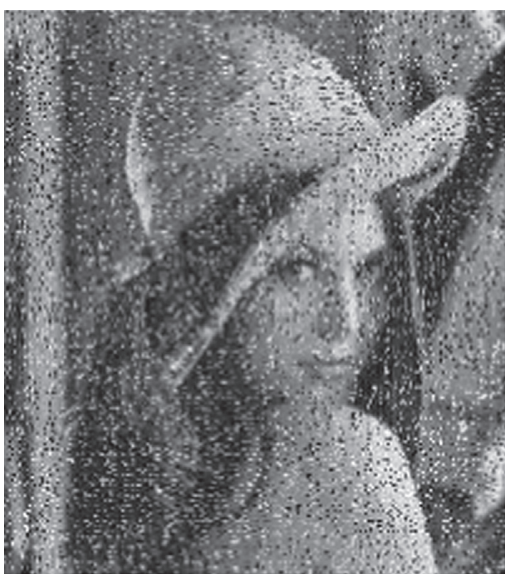

(a)

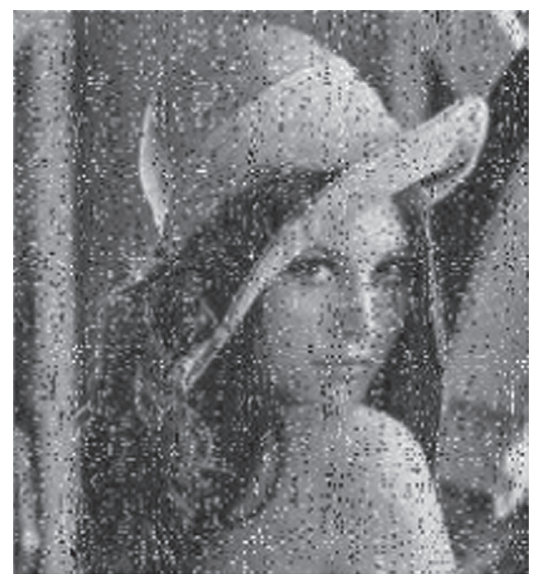

(b)

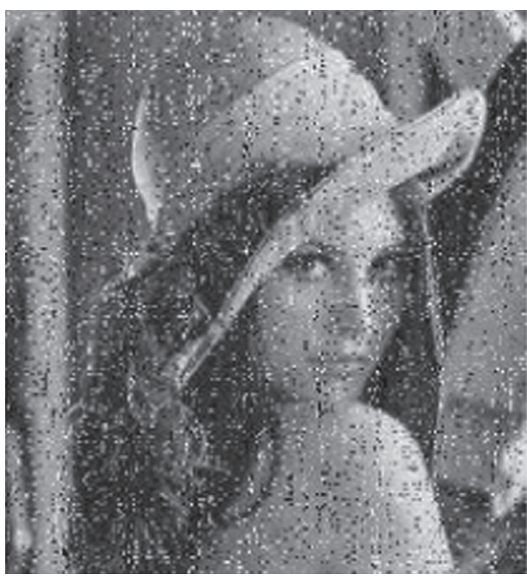

(c)

FIGURE 3: Recovered RS-coded image (transmitted over Rician fading channel-added Gaussian noise). (a) $\mathrm{SNR}=-2 \mathrm{~dB}$; PSNR $=32.87$. (b) $\mathrm{SNR}=-1 \mathrm{~dB}$; PSNR $=34.62$. (c) $\mathrm{SNR}=0 \mathrm{~dB} ; \mathrm{PSNR}=36.72$.

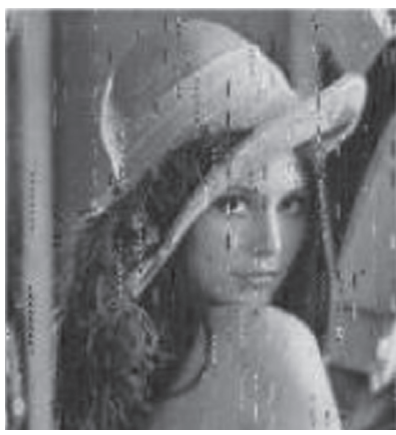

(a)

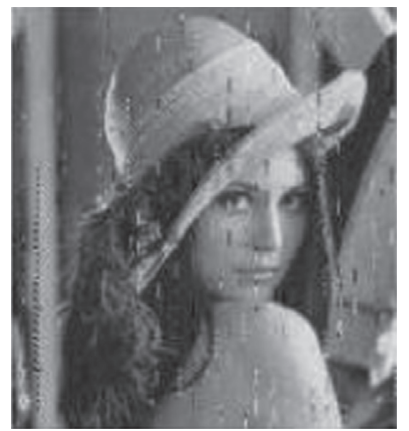

(b)

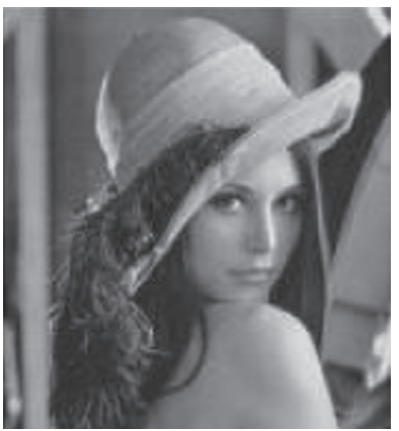

(c)

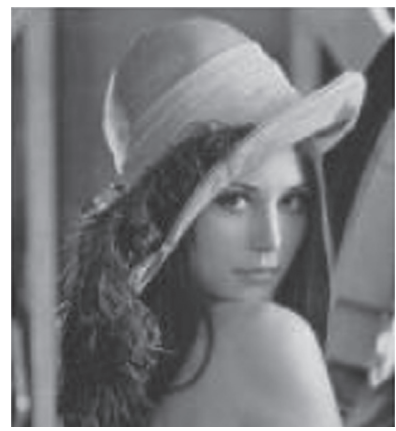

(d)

FIgURe 4: Recovered Polar-coded image and RS-coded image (with periodic strong pulse jamming). (a) JSR = $5 \mathrm{~dB}$; PSNR $=38.5$. (b) $\mathrm{JSR}=10 \mathrm{~dB} ; \mathrm{PSNR}=38.4$. (c) $\mathrm{JSR}=5 \mathrm{~dB} ; \mathrm{PSNR}=$ inf. (d) $\mathrm{JSR}=10 \mathrm{~dB} ; \mathrm{PSNR}=$ inf. 


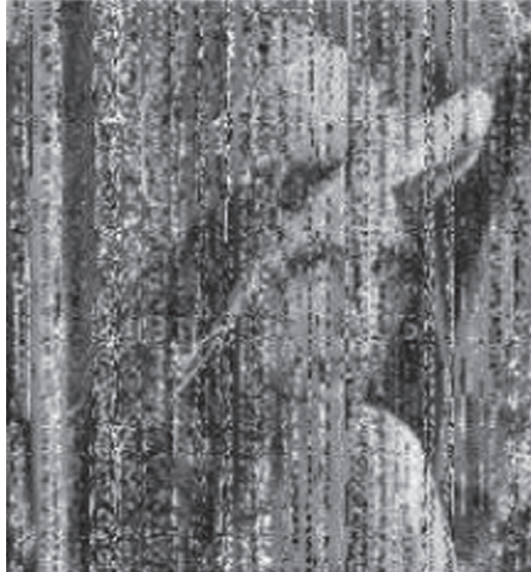

(a)

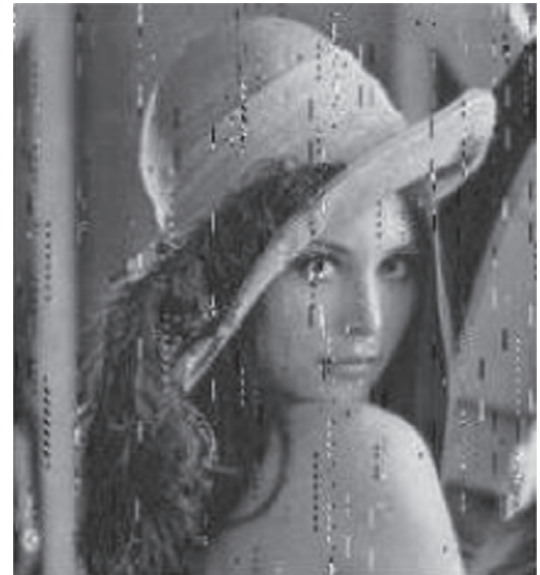

(b)

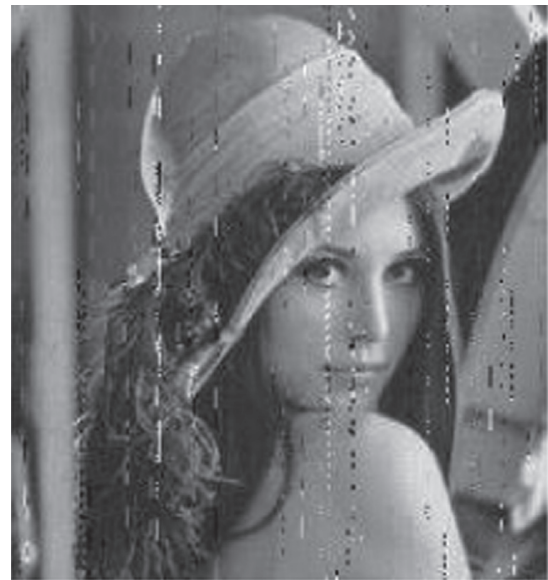

(c)

Figure 5: Recovered Polar-coded images (transmitted over fading channel-added periodic strong pulse jamming). (a) SNR $=-2$; Psnr $=30.6215$. (b) $\mathrm{SNR}=0 ; \mathrm{psnr}=38.1989$. (c) $\mathrm{SNR}=2 ; \mathrm{psnr}=39.5724$.

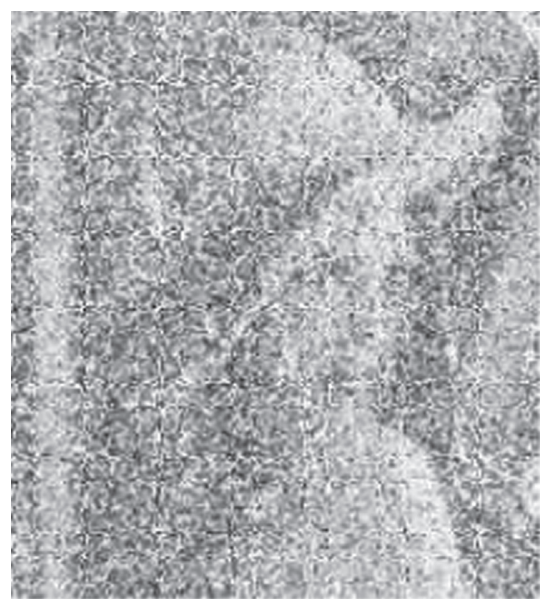

(a)

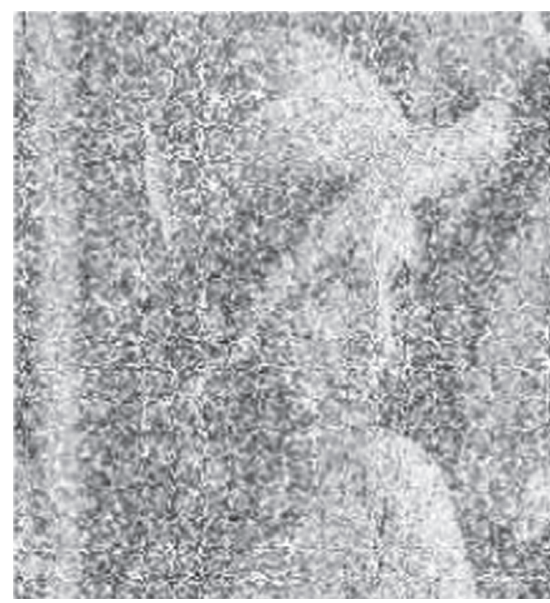

(b)

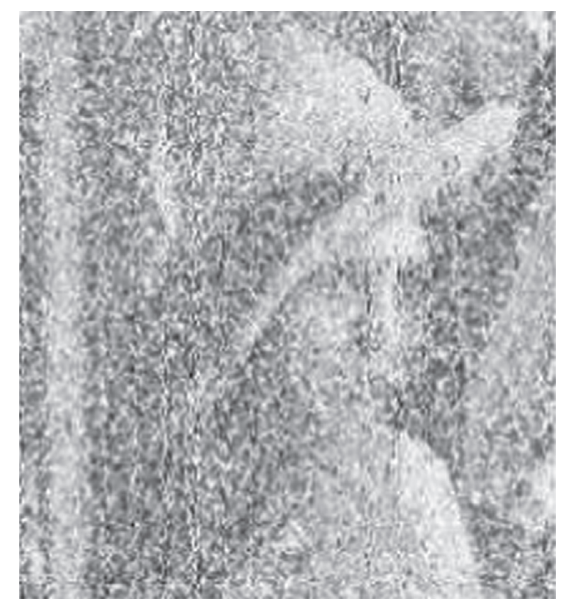

(c)

FIGURE 6: Received RS-coded images (transmitted over fading channel-added periodic strong pulse jamming). (a) $\mathrm{SNR}=-2 ; \mathrm{PSNR}=26.48$. (b) $\mathrm{SNR}=0 ; \mathrm{PSNR}=28.79$. (c) $\mathrm{SNR}=2 ; \mathrm{PSNR}=30.4$.

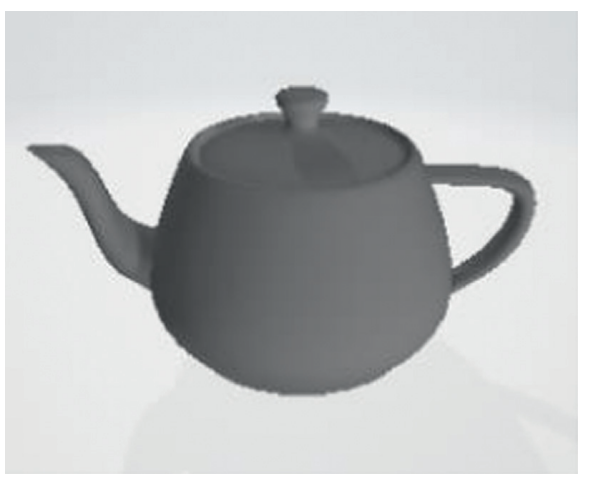

(a)

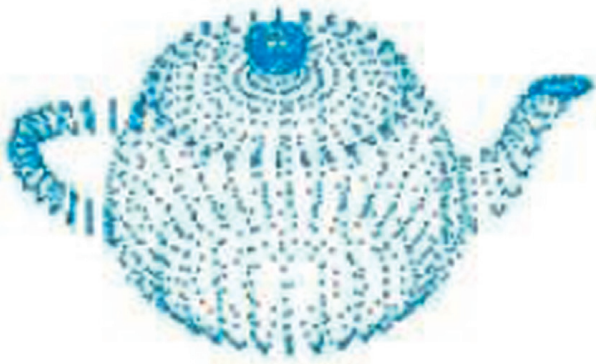

(b)

FIgURE 7: 3D image of the teapot. (a) Original obj image. (b) Extracted 3D point cloud. 


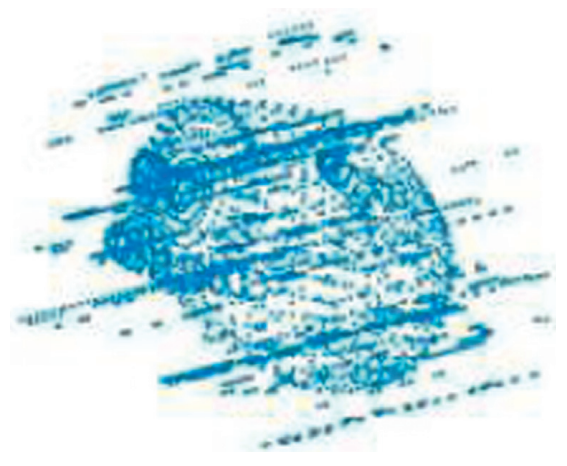

(a)

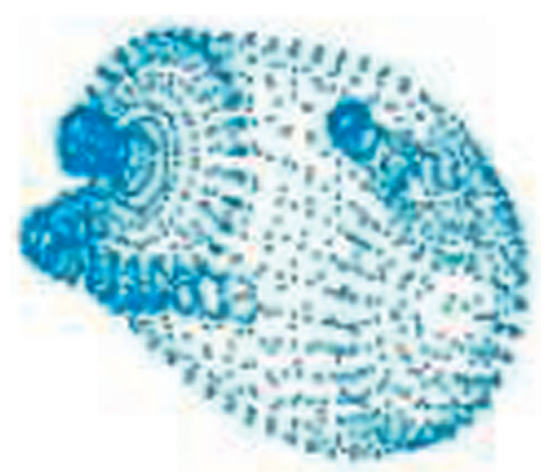

(b)

FIGURE 8: Reconstructed 3D point cloud (Polar-coded system with fading channel and AWGN noise). (a) $\mathrm{SNR}=0 \mathrm{~dB}$; (b) $\mathrm{SNR}=2 \mathrm{~dB}$.

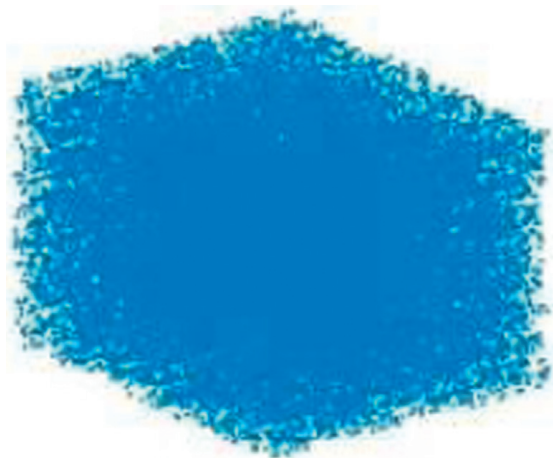

(a)

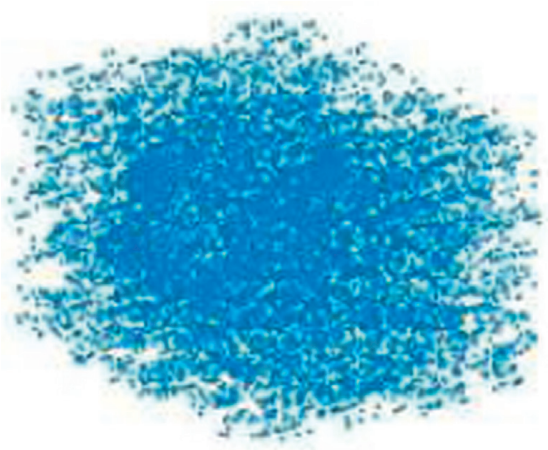

(b)

FIGURE 9: Reconstructed 3D point cloud (RS-coded system with fading channel and AWGN noise). (a) $\mathrm{SNR}=0 \mathrm{~dB}$; (b) SNR = $2 \mathrm{~dB}$.

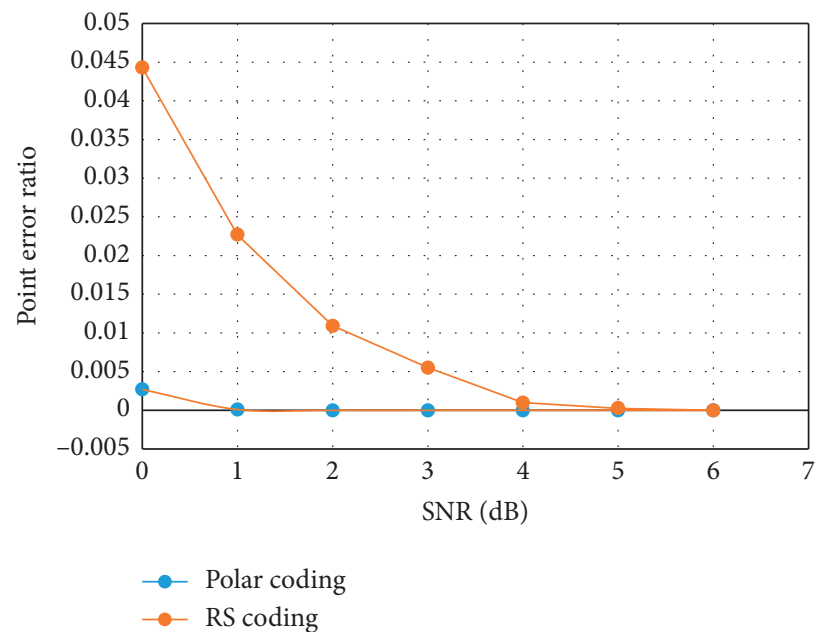

FIgURe 10: Point error ratio versus SNR (Polar/RS system with fading channel and AWGN noise).

can be seen from Figure 14, a wide pulse width (pw) leads to a worse image quality (a large $M$ value).

It can be seen from Table 1 that when jamming pulse width is within a certain limit (less than $51.2 \mu \mathrm{s}$ ), strong jamming pulses have no effect on the quality of the received RS-coded point cloud image. Compared to Polar, it indicates that the performance of RS code scheme is better than that of Polar code scheme on antistrong pulse jamming. 


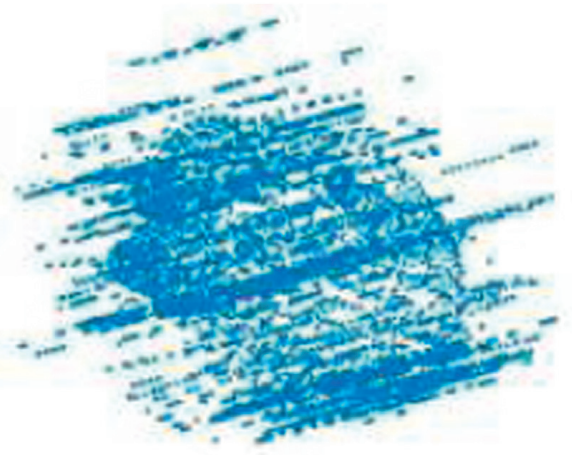

(a)

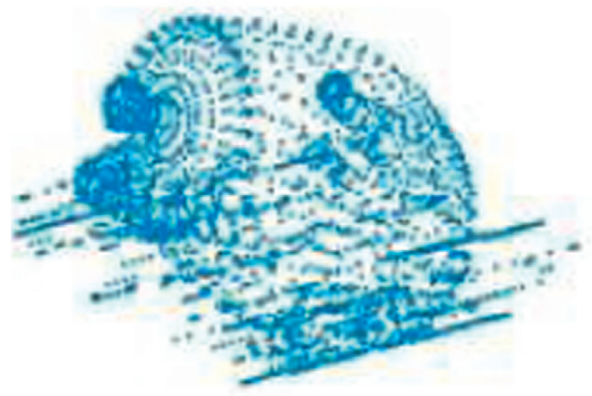

(c)

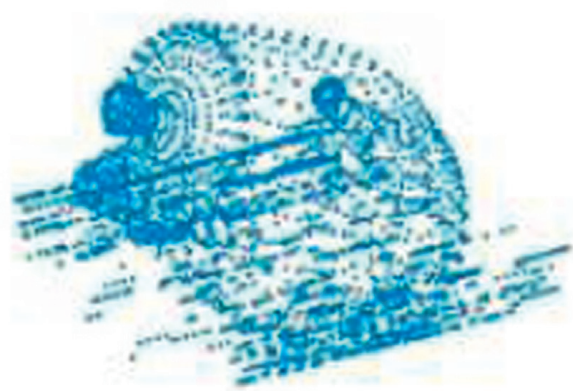

(b)

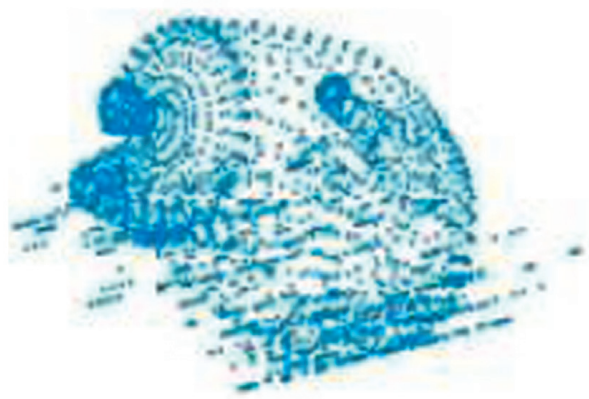

(d)

FIGURE 11: Reconstructed 3D point cloud (Polar-coded system with fading channel and AWGN noise and periodic strong pulse jamming). (a) $\mathrm{SNR}=0(M=4069)$; (b) $\mathrm{SNR}=2(M=1100)$; (c) SNR=4 $(M=1052)$; (d) SNR=6 $(M=744)$.

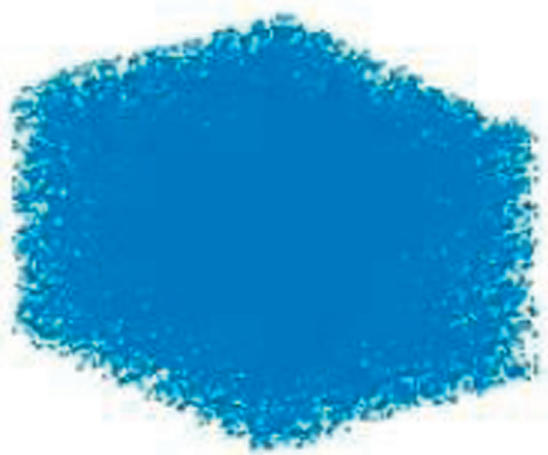

(a)

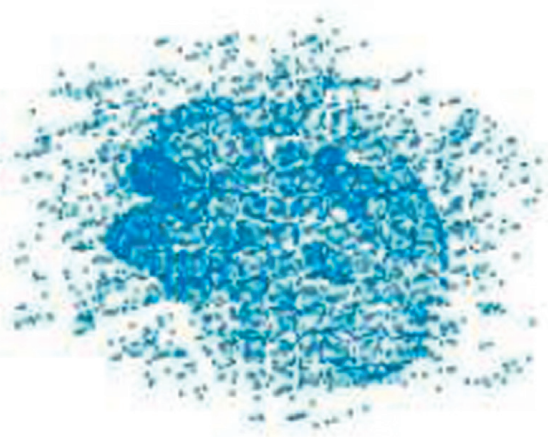

(c)

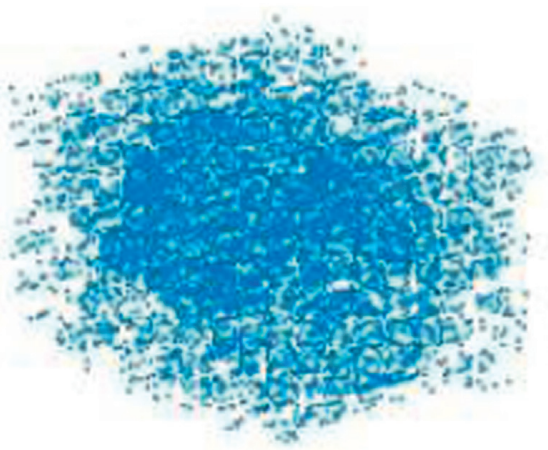

(b)

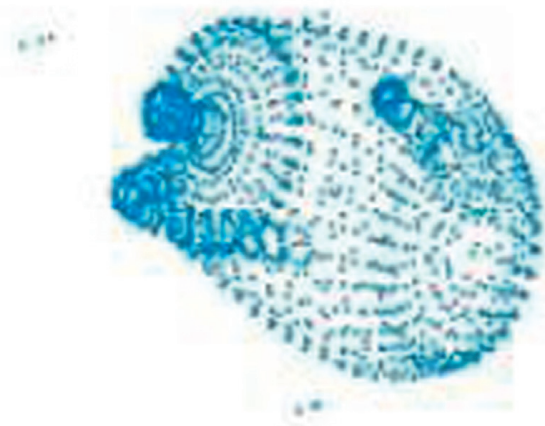

(d)

FIGURE 12: Reconstructed 3D point cloud (RS-coded system with fading channel and AWGN noise and periodic strong pulse jamming). (a) $\mathrm{SNR}=0(M=37387)$; (b) SNR = $2(M=8820)$; (c) $\mathrm{SNR}=4(M=1486)$; (d) SNR=6 $(M=30)$. 


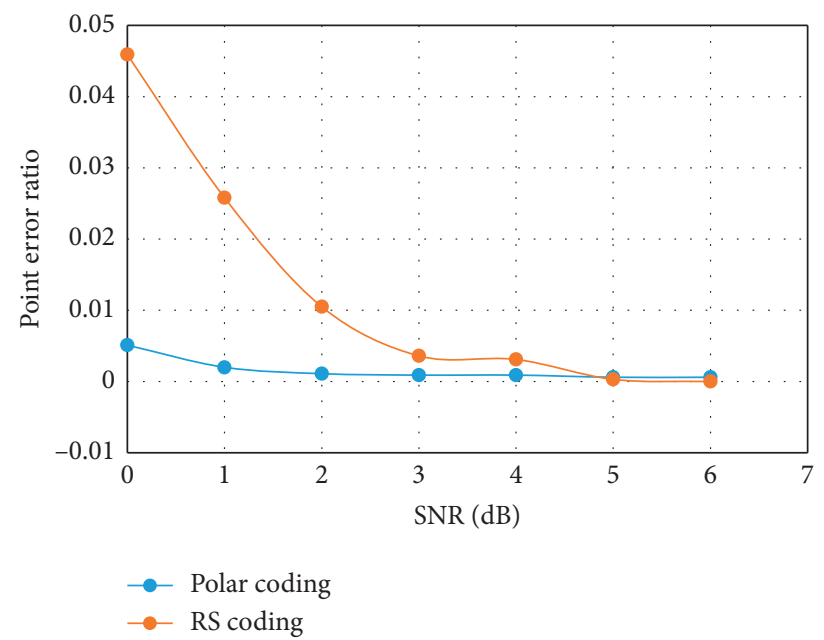

Figure 13: Point error ratio versus SNR (Polar/RS system with fading channel, AWGN noise, and strong pulse jamming).

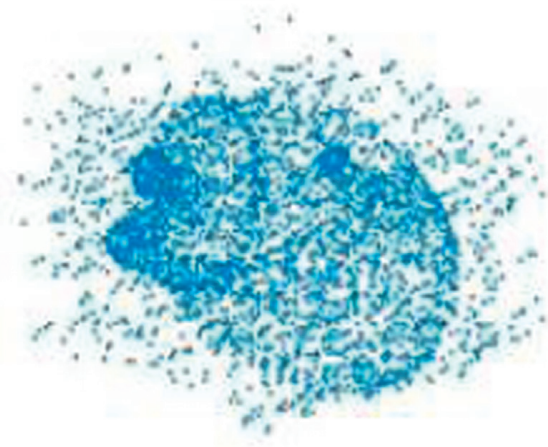

(a)

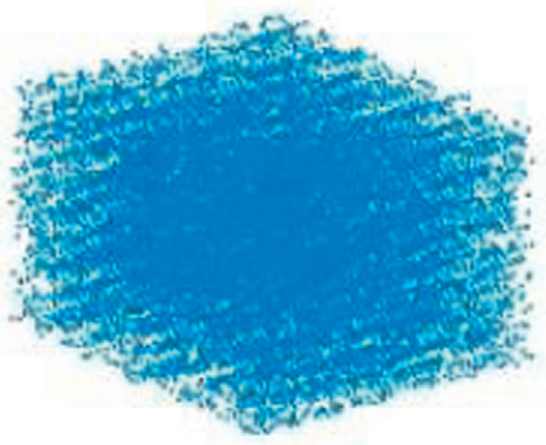

(b)

Figure 14: Recovered Polar-coded 3D point cloud (with periodic strong pulse jamming). (a) pw $=12.8 \mu \mathrm{s}(M=1572)$; (b) pw $=51.2 \mu \mathrm{s}$ $(M=18336)$.

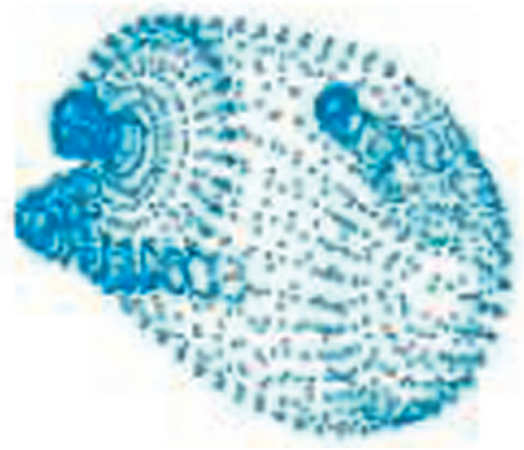

(a)

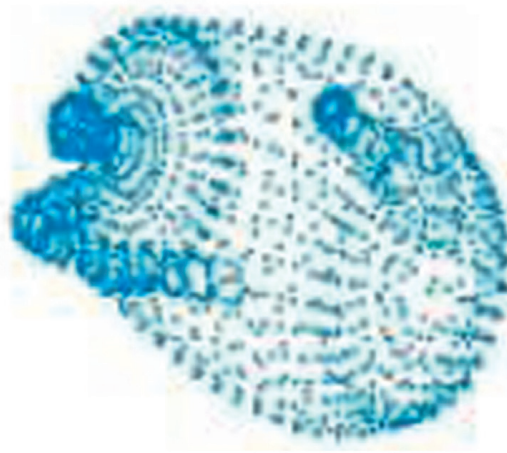

(b)

Figure 15: Recovered RS-coded 3D point cloud (with periodic strong pulse jamming). (a) $\mathrm{Pw}=12.8 \mu \mathrm{s}(M=0)$; (b) pw=51.2 $\mu$ s $(M=0)$. 
TABLE 1: Multimodel comparison.

\begin{tabular}{lcc}
\hline Pulse width $(\mu \mathrm{s})$ & Error points $(M)$ (polar code) & Error points $(M)(\mathrm{RS}$ code) \\
\hline $6.4(32$ symbol $)$ & 373 & 0 \\
$12.8(64$ symbol $)$ & 1572 & 0 \\
$25.6(128$ symbol $)$ & 6299 & 0 \\
$51.2(256$ symbol $)$ & 18336 & 0 \\
$76.8(384$ symbol $)$ & 28216 & 106745 \\
$102.4(512$ symbol $)$ & 42714 & 140733 \\
\hline
\end{tabular}

\section{Conclusion}

We established an image transmission system model based on Polar/RS code scheme and then tested its performances on combating channel fading, additive Gaussian white noise, and periodic pulse jamming by transmitting $2 \mathrm{D}$ image and 3D point cloud image, respectively. Simulation results indicate that Polar-coded system provides better performances on antifading and anti-AWGN noise than RS system does, whereas RS system performs well on antiperiodic pulse jamming. Simulation results also show that the improvement of JSR of strong jamming pulse has a little impact on received image quality, while the increase in width of strong jamming pulse degrades the $3 \mathrm{D}$ point cloud quality evidently.

\section{Data Availability}

The .xls data used to support the findings of this study are available from the corresponding author upon request.

\section{Conflicts of Interest}

The authors declare that they have no conflicts of interest.

\section{Acknowledgments}

This work was funded by the National Natural Science Foundation of China, under Grant nos. 51874300 and 61801270, Shanxi Provincial People's Government Jointly Funded Project of China for Coal Base and Low Carbon, under Grant no. U1510115, and the Open Research Fund of Key Laboratory of Wireless Sensor Network and Communication, Shanghai Institute of Microsystem and Information Technology, Chinese Academy of Sciences, under Grant nos. 20190902 and 20190913.

\section{References}

[1] S. Siebert and J. Teizer, "Mobile 3D mapping for surveying earthwork projects using an Unmanned Aerial Vehicle (UAV) system," Automation in Construction, vol. 41, pp. 1-14, 2014.

[2] L. Comba, A. Biglia, D. Ricauda Aimonino, and P. Gay, "Unsupervised detection of vineyards by 3D point-cloud UAV photogrammetry for precision agriculture," Computers and Electronics in Agriculture, vol. 155, pp. 84-95, 2018.

[3] D. W. Matolak, "3D Air-X UAV communications: challenges and channel modeling," in Proceedings of the International Workshop on Communication Technologies for Vehicles, Lecture Notes in Computer Science, Moreno, CA, USA, May 2018.
[4] H. S. Silvaa, M. S. Alencarb, W. J. L. Queirozc, D. B. T. Almeida, and F. Madeiro, "Bit error probability of M-QAM under $\eta-\mu$ or $\kappa-\mu$ fading and impulsive noise gated by a signal characterized by a Markov process or Poisson process," Digital Signal Processing, vol. 100, pp. 1-14, 2020.

[5] Y. Li, W. Wang, H. Gao et al., "Air-to-ground 3D channel modeling for UAV based on Gauss-Markov mobile model," AEU-International Journal of Electronics and Communications, vol. 114, Article ID 152995, 2020.

[6] S. Zhao, P. Shi, and B. Wang, "Polar codes and its application in speech communication," in Proceedings of the International Conference on Wireless Communications and Signal Processing (WCSP), pp. 1-4, IEEE, Nanjing, China, November 2011.

[7] A. Mishra, K. Sharma, and A. De, "Quality image transmission through AWGN channel using polar codes," International Journal of Computer Science and Telecommunications, vol. 5, no. 1, 2014.

[8] P. Shi, Z. Zhifang, and L. Gong, "Studies on performance of polar coded image transmission," Journal of Nanjing University of Post \& Telecommunication (Natural Science), vol. 34, no. 2, pp. 65-71, 2014.

[9] H. Fan, S. Zhao, and B. Zheng, "Construction and analyses corresponding performance of polar codesin rician channels," Journal of Signal Processing, vol. 31, no. 9, pp. 1062-1066, 2015.

[10] M. A. M. Mohamed El-Bendary, A. E. Abou El-Azm, N. A. ElFishawy et al., "JPEG image transmission over mobile network with an efficient channel coding and interleaving," International Journal of Electronics, vol. 99, no. 11, Article ID 1491518, 2012.

[11] C. Yang, Z. Wang, W. He, and Z. Li, "Development of a fast transmission method for 3D point cloud," Multimedia Tools and Applications, vol. 77, no. 19, pp. 25369-25387, 2018.

[12] D. Wu, Y. Li, and Y. Sun, "Construction and block error rate analysis of polar codes over AWGN channel based on Gaussian approximation," IEEE Communications Letters, vol. 18, no. 7, pp. 1099-1102, 2014.

[13] S. Kahraman and M. E. Celebi, "Code based efficient maximum-likelihood decoding of short polar codes," in Proceedings of the 2012 IEEE International Symposium on Information Theory Proceedings, pp. 1967-1971, IEEE, Cambridge. Massachusetts, USA, July 2012.

[14] I. Tal and A. Vardv, "List decoding of polar codes," in Proceedings of the 2011 IEEE International Symposium on Information Theory Proceedings, IEEE, St. Petersburg, Russia, July 2011.

[15] K. Chen, K. Niu, and J. R. Lin, "Improved successive cancellation decoding of polar codes," Electronics Letters, vol. 61, no. 8, pp. 3100-3107, 2012. 